\title{
Moralistes et remarqueurs
}

\author{
Éric Tourrette
}

\section{(2) OpenEdition}

Journals

Édition électronique

URL : http://journals.openedition.org/studifrancesi/278

DOI : 10.4000/studifrancesi.278

ISSN : 2427-5856

\section{Éditeur}

Rosenberg \& Sellier

\section{Édition imprimée}

Date de publication : 1 avril 2015

Pagination : 22-34

ISSN : 0039-2944

\section{Référence électronique}

Éric Tourrette, « Moralistes et remarqueurs », Studi Francesi [En ligne], 175 (LIX | I) | 2015, mis en ligne le 01 avril 2016, consulté le 18 septembre 2020. URL : http://journals.openedition.org/studifrancesi/ 278 ; DOI : https://doi.org/10.4000/studifrancesi.278

\section{(c) (i) (2) $\Theta$}

Studi Francesi è distribuita con Licenza Creative Commons Attribuzione - Non commerciale - Non opere derivate 4.0 Internazionale. 


\section{Moralistes et remarqueurs}

\section{Abstract}

In the 17th century, short forms are used in two parallel fields, which appear to be different and have separate specialisations: the moral analysis, such as in La Rochefoucauld or La Bruyère; and the grammatical analysis, as in Vaugelas or Bouhours. Few scholars have compared these two categories of authors, the moralists and the remarqueurs. This paper aims to outline the existing correlations in two ways. On one hand, there are some «internal» convergences in the authors' discourses: the topics, the arguments they put forward, the words they utilise. On the other hand, the «external» convergences, often surprisingly precise, are not uncommon in comments by the specialists, to the point that the critical arguments give the impression of being duplicated.

«Moralistes et remarqueurs»: ce titre suscitera sans doute une double réaction chez le lecteur. D'une part, ce dernier reconnaîtra aisément un certain nombre de traits communs aux deux familles d'auteurs, à commencer par la notion cruciale de forme brève, assurément transversale: ainsi, Vaugelas et La Bruyère emploient tous deux le mot de «remarques» pour désigner leurs productions respectives, assumant par là une posture (un ethos) d'humbles observateurs du réel, aussi discrets que prudents. L'abbé de Bellegarde symbolise exemplairement, à lui seul, les sourdes affinités entre ces deux prestigieux prédécesseurs, puisqu'il les imite conjointement, quasi au même moment, dans deux volumes distincts dont les titres se recoupent étroitement, à travers l'étiquette générique de «réflexions» et la coordination des thèmes traités, comme si les deux entreprises étaient étroitement mêlées dans son esprit: aux Reflexions sur l'élegance et la politesse du stile semblent faire écho les Reflexions sur le ridicule, et sur les moyens de l'éviter ${ }^{1}$. On parle aussi bien de «faiseurs de remarques» que de «faiseur[s] de maximes» ${ }^{2}$. Il ne serait pas difficile de montrer qu'un remarqueur peut aborder ponctuellement les questions de civilité, et qu'un moraliste ne dédaigne pas les questions linguistiques. Et il arrive, dans les commentaires critiques, que les mots se croisent, pour former des chiasmes collectifs, suggestifs d'une vraie complémentarité: d'un côté on évoque «le côté moraliste du livre de Vaugelas», de l'autre on voit en La Bruyère un «grammairien du réel» ${ }^{3}$. De tels faits sont moins anecdotiques qu'il n'y paraît: pour éclatés qu'ils soient, ils convergent pour suggérer une cohésion profonde, qu'on aurait spontanément tendance, aujourd'hui, à méconnaître ou à sous-estimer.

Mais s'imposera aussi, d'autre part et inversement, le sentiment que ces deux

(1) Respectivement: Paris, André Pralard, 1695; Paris, Jean Guignard, 1696.

(2) ABBÉ DE Bellegarde, Reflexions sur l'élegance et la politesse du stile cit., préface; lettre anonyme de 1663, dans La RocheFoucauld, Maximes, éd. J. Rohou, Paris, Livre de Poche, 1991, p. 301.
(3) K.A. Отт, La notion du "bon usage" dans les Remarques de Vaugelas, «C.A.I.E.F.» n. 14, 1962, p. 94; E. TourretTe, Les Formes brèves de la description morale: Quatrains, maximes, remarques, Paris, Champion, 2008, p. 303. 
domaines de recherche ne sont pas vraiment du même ordre, et sont peu appelés à se croiser vraiment: car la coupure est forte entre les spécialistes respectifs de l'histoire de la littérature et de l'histoire de la grammaire, et le caractère constitutivement compartimenté de l'Université ne favorise guère de telles rencontres intellectuelles. La recherche dix-septiémiste - et le constat peut sans doute être élargi - n'est pas le lieu de la porosité, mais de l'étanchéité des savoirs; car il est déjà si difficile de connaître un peu un domaine de recherche, que l'idée même de s'intéresser simultanément à un autre semble pour le moins déraisonnable. Et nul ne songe à contester que ni les compétences requises, ni les méthodes critiques ne sont rigoureusement superposables: considérée comme un document sur l'état de la langue en 1647, une remarque de Vaugelas peut être évaluée scientifiquement, et donner lieu à un jugement sur sa fiabilité ou son inexactitude; l'éditeur des Remarques n'hésitera donc pas à signaler, au nom du savoir, la fausseté objective d'une étymologie ou d'une observation syntaxique. Mais il n'y a évidemment rien de tel pour les questions autrement troubles et abstraites qu'aborde un La Rochefoucauld: un philosophe peut toujours exprimer son accord ou son désaccord de principe avec telle ou telle analyse psychologique ponctuelle, mais nul ne s'aviserait sérieusement de déclarer «vraie» ou «fausse» une maxime, dont l'appréciation se fera plutôt en vertu d'idéaux esthétiques. La fascination littéraire qu'on éprouve pour La Rochefoucauld n'est assurément pas analogue à l'intérêt scientifique que suscite Vaugelas, même s'il est évident que celui-ci sait écrire, et que celui-là sait observer.

L'Université compte donc en son sein un certain nombre de chercheurs qui travaillent sur les formes brèves de l'analyse morale, et un certain nombre de chercheurs qui travaillent sur les formes brèves de l'analyse linguistique: ce ne sont pas les mêmes personnes, et l'on peut dire, sans trop s'avancer, que personne n'étudie, de façon transversale, l'ensemble des formes brèves de l'Âge classique - tâche démesurée s'il en est, voire irréalisable. Cette situation, imposée par les contraintes scientifiques et institutionnelles, crée un curieux dédoublement des discours, dont on ne prend nettement conscience qu'avec un peu de recul, à la manière des moralistes, qu'on sait toujours désireux de situer le juste «point de perspective» d'où peut s'observer un spectacle. Des échos parfois étroits se manifestent entre ces deux entreprises conjointes, mais nul n'a vraiment le loisir de s'en préoccuper: on songe à des lignes parallèles, c'est-à-dire à la fois toujours analogues et toujours distinctes. Un lien spéculaire, parfois vaguement aperçu ou sourdement pressenti, mais rarement décrit en détail ${ }^{4}$, unit moralistes et remarqueurs, opérant ainsi l'unification profonde de la pensée de l'Âge classique, quelle que soit la matière (verbale ou morale) étudiée. De même, les commentaires des spécialistes respectifs de ces deux types de formes brèves se recoupent souvent avec une étonnante précision, généralement à l'insu des principaux concernés: ce sont, de part et d'autre, les mêmes formules, les mêmes arguments, les mêmes conclusions.

Le présent article n'a d'autre ambition que de dégager quelques-uns de ces points de rencontre, en associant deux démarches complémentaires: identifier les convergences «internes» entre les analyses respectives des moralistes et des remarqueurs (dans leur méthode, leur langage, leur attitude, etc.), mais aussi dégager des recoupements «externes» entre les commentaires des critiques littéraires et des historiens de la grammaire. Deux séries de topö̈, premiers ou seconds, se manifestent ainsi: ceux qui sont cultivés par les auteurs, ceux qui sont forgés de toutes pièces par

(4) Voir toutefois, pour un premier survol de la question: É. TourretTe, La Bruyère grammairien, 
le discours critique. Le discours des auteurs et le discours sur les auteurs seront ici dissociés pour la seule commodité de la présentation. Mais on conçoit aisément qu'il n'y a pas de séparation tranchée entre ces deux approches, et qu'il serait par trop artificiel de creuser l'écart entre l'objet d'étude et les représentations qu'il suscite, celui-là pouvant difficilement s'atteindre sans passer par celles-ci.

Il faut encore préciser, au risque de surprendre, que le lecteur trouvera ici peu de commentaires: ce qu'on lui propose, c'est plutôt un parcours citationnel, un dossier formé de simples textes juxtaposés en vue d'accuser les affinités et d'appeler les prolongements interprétatifs, c'est-à-dire des matériaux pour un comparatif virtuel. On a toutefois évité de multiplier les citations analogues - ce qui eût été aisé, tant les discours collectifs sont stables; on en livre le moins possible, en les choisissant pour leur représentativité.

\section{Convergences internes}

Les agréments du désordre

D'un moraliste à l'autre, d'un remarqueur à l'autre, les mots employés dans les titres ne sont pas toujours les mêmes, mais le pluriel - indice grammatical de l'éclatement constitutif du genre - semble quasi systématique: ce ne sont, de part et d'autre, que remarques, caractères, réflexions, maximes, observations, considérations, doutes, commentaires... Le singulier construirait la représentation d'un objet stable et homogène, quand le pluriel reconnaît et revendique l'éparpillement d'une parole et d'une pensée, souplement conduites par la libre association et l'art du rebond inopiné. Mais la forme brève est toujours suspecte d'inconsistance ou de facilité: l'histoire de la réception du genre est celle d'un long «procès», constamment relancé sur les mêmes griefs ${ }^{5}$. Ce que C. Rosso décrit au sujet des moralistes peut aisément s'appliquer aux remarqueurs: tous partagent une tenace mauvaise réputation, que désavouent bien entendu ceux qui les lisent, mais que propagent ceux, autrement nombreux, qui ne les lisent pas. Aussi le moraliste ou le remarqueur s'ingénie-t-il souvent, dans un élément paratextuel quelconque (préface, lettre, avertissement...), à rendre compte de la présentation éclatée qu'il adopte: tout se passe comme si le choix de la forme brève n'allait jamais de soi, et comme s'il fallait toujours établir au préalable sa légitimité. Les arguments allégués sont toujours, grossièrement, les mêmes: ils forment une topique collective, largement inspirée des préceptes de la civilité, où dominent le souci de l'agrément du lecteur et le refus d'une présentation systématique perçue comme fastidieuse. La forme brève s'affiche, idéalement, comme un refus de tout ce qui sent le pédantisme et la poussière des bibliothèques.

Moralistes et remarqueurs présentent donc volontiers leurs œuvres respectives comme entièrement dépourvues d'ordre, ce qui est évidemment excessif. L'ordre de succession, certes, est toujours souple: «le hasard des rencontres dicte assez souvent l'ordre capricieux d'exposition»; «ces ouvrages ont tous pour point commun le refus de la continuité méthodique» ${ }^{6}$. Mais souvent il n'est pas arbitraire: «plusieurs observations consécutives ont des liens»; «les maximes sont regroupées en thèmes plus ou moins homogènes» ${ }^{7}$.

(5) Voir C. Rosso, Procès à La Rochefoucauld et à la maxime, Pise, Goliardica, 1986.

(6) Ph. Caron, «Postface», dans Ph. Caron (dir.), Les Remarqueurs sur la langue française $d u$ XVI siècle à nos jours, Rennes, P.U.R., 2004, p. 396; B. Parmentier, Le Siècle des moralistes, Paris, Seuil,
2000, p. 17.

(7) W. Ayres-BennetT et M. SeijIDO, Remarques et observations sur la langue française: Histoire et évolution d'un genre, Paris, Classiques Garnier, 2011, p. 44; G. MinoIs, La Rochefoucauld, Paris, Tallandier, 2007, p. 448. 
Vaugelas suggère ainsi, par le biais de l'enthymème, que le désordre des Remarques devrait interdire toute beauté à l'entreprise, et La Rochefoucauld tient à peu près le même langage: «on trouuera estrange, que ie n'aye obserué aucun ordre en ces Remarques, n'y ayant rien de si beau ny de si necessaire que l'ordre en toutes choses»; «pour ce qui est de l'ordre de ces réflexions, [...] il était difficile d'y en observer»8. Vaugelas continue en rejetant deux ordres virtuellement envisageables pour le volume. L'ordre méthodique des parties du discours ne serait accessible qu'aux lecteurs formés à la grammaire latine, contrevenant ainsi à l'idéal d'un ouvrage agréable pour les honnêtes gens: et Vaugelas de rappeler qu'il n'entend pas rédiger une «Grammaire» en bonne et due forme. Quant à l'ordre purement conventionnel de l'alphabet, il ne ferait finalement qu'accroître la confusion, en opérant des juxtapositions arbitraires de matières dissemblables; en outre, il ferait double emploi avec la table des matières qui clôt de toute façon le volume et qui en facilite la consultation: «la Table [...] reduit à l'ordre de l'Ảlphabet toutes les choses principales qu'elles contiennent»; «il y en $[\mathrm{a}]$ plusieurs sur un même sujet, [...] on les trouvera dans la table»`.

Pour décrire ce que nous appelons, anachroniquement, des «formes brèves», les auteurs du XVII ${ }^{\mathrm{e}}$ siècle s'accordent à employer le mot «détaché», autrement dit ils sont moins sensibles à la concision de chaque paragraphe qu'à l'absence de lien (anaphorique) avec l'entourage cotextuel immédiat. La forme brève est plutôt une forme coupée, elle se définit par son isolement plutôt que par son volume intrinsèque: «des Remarques, qui sont toutes destachées l'vne de l'autre, \& dont l'intelligence ne depend nullement, ny de celles qui precedent, ny de celles qui suiuent»; «il s'en trouve un [de traité] sous le titre de Proverbes, c'est-à-dire de pièces détachées, comme des réflexions ou des remarques» ${ }^{10}$.

La forme brève émiette donc l'analyse: c'est une pensée éparpillée qui se déploie de prime abord, et c'est au lecteur d'en retrouver, activement, la cohérence souterraine. Ce jeu de puzzle est présenté comme virtuellement délectable pour un public d'honnêtes gens: «il y a vne certaine confusion qui a ses charmes»; «ce désordre néanmoins a ses grâces» ${ }^{11}$. L'imprévisible, la légèreté, la souplesse, la plume sautillante et désinvolte qui erre au hasard de l'inspiration, sont autant d'idéaux culturels et esthétiques qui renvoient au grand modèle de la conversation mondaine, ennemie de toute contrainte: «l'agrément d'un entretien familier à bâtons rompus»; «l'allure plaisante, à bâtons rompus, des conversations familières» ${ }^{12}$.

Or, l'un des dogmes essentiels de la conversation honnête est le refus du ton d'autorité, indice d'un orgueil coupable et d'une volonté de dominer l'interlocuteur. Ce précepte recoupe le grand topos de la modestie affichée, indispensable pour opérer la captatio benevolentiae selon l'Empire rhétorique. Aussi la posture du législateur n'est-elle alléguée que sur le mode du déni explicite: «ce ne sont pas icy des Loix que ie fais pour nostre langue de mon authorité priuée»; «je n'ai ni assez d'autorité ni assez de génie pour faire le législateur» ${ }^{13}$.

(8) Vaugelas, Remarques sur la langue française [1647], éd. J. Streicher, Paris, Droz, 1934, préface, XII; La Rochefoucauld, op. cit., «Le libraire au lecteur», p. 74.

(9) Vaugelas, ibid.; La Rochefoucauld, ibid.

(10) Vaugelas, ibid;; La Bruyère, Les Caractères, «Discours sur Théophraste», dans J. LAFOND (dir.), Moralistes du XVII siecle, Paris, Laffont, 1992, p. 667.
(11) Vaugelas, ibid.; La Chapelle-Bessé, «Discours sur les Maximes», dans La RochefoucAuld, op. cit., p. 275.

(12) R. Lagane, «Notice», dans Vaugelas, Remarques sur la langue française, Paris, Larousse, 1969, p. 12; B. PARMENTIER, op. cit., p. 19.

(13) Vaugelas, op. cit., préface, I; La Bruyère, op. cit., préface, p. 695. 
L'esprit de discernement

Le «discernement» est l'un des concepts essentiels, et peut-être même le concept central, pour décrire la démarche des moralistes classiques: eux-mêmes le revendiquent explicitement, et l'on se condamne à mal comprendre leur pensée, leur écriture et leur méthode si on l'oublie un instant. C'est un art de voir nettement un spectacle, mais aussi de trier les référents, et d'établir de minutieuses typologies, fondées sur des distinctions d'autant plus stimulantes qu'elles sont plus subtiles: la finesse hyperbolique, la nuance infiniment ténue, la saisie microscopique sont ici de mise. On peut dire précisément la même chose de l'entreprise des remarqueurs: «[le livre] de Vaugelas brille par l'esprit de discernement»; «le principe de discernement hante littéralement l'univers des Caractères» ${ }^{14}$. La notion de «préciosité» n'est que l'hyperbole ou la caricature du discernement: «penchant fâcheux à la préciosité»; «l'œuvre qu'avaient déjà commencée les Précieuses, La Bruyère l'a poursuivie avec un souci remarquable» ${ }^{15}$.

C'est particulièrement visible dans l'ample réflexion sur les synonymes; il y a souvent chez les remarqueurs un acharnement exalté à repérer - ou à construire, voire à imaginer - une nuance ingénieuse entre les concurrents, au nom de l'exactitude et au risque de l'artifice, d'où le recours à une procédure de différenciation que la rhétorique nomme paradiastole: «Malheureux, miserable. Ces deux mots se ressemblent extrêmement: ils ne laissent pas d'avoir quelque chose de particulier l'un \& l'autre»; «bien que la sincérité et la confiance aient du rapport, elles sont néanmoins différentes en plusieurs choses» ${ }^{16}$.

La nécessité d'établir une nuance s'impose plus encore quand aux parentés du signifié s'ajoutent les parentés du signifiant, autrement dit quand les synonymes se trouvent être, de surcroît, des paronymes:

Estre docte, c'est estre véritablement savant et habile. Estre docteur, c'est non seulement estre habile homme, mais avoir donné certaines preuves de sa science par lesquelles on ait obtenu ce titre. Il faut néanmoins avouer qu'aujourd'huy le mot de docteur est fort au-dessous de celuy de docte.

Un homme à la Cour, et souvent à la Ville, qui a un long manteau de soie ou de drap de Hollande, une ceinture large et placée haut sur l'estomac, [...] qui avec cela se souvient de quelques distinctions métaphysiques, explique ce que c'est que la lumière de gloire, et sait précisément comment l'on voit Dieu, cela s'appelle un docteur. Une personne humble, qui est ensevelie dans le cabinet, qui a médité, cherché, consulté, confronté, lu ou écrit pendant toute sa vie, est un homme docte ${ }^{17}$.

Parfois aussi, les remarqueurs doivent se résoudre à reconnaître que deux mots peuvent s'utiliser «indifféremment»: ce mot, récurrent sous leur plume, sonne alors comme un constat d'échec, en ce qu'il dit les limites du rêve d'analyse et de différenciation. Peut-être la nuance existe-t-elle, virtuellement: mais leur propre regard, pour affûté qu'il soit, ne parvient pas à la déceler. Idéalement, tout devrait être séparé en sphères bien distinctes: chevauchements, recoupements ou empiétements ont ici

(14) E. Moncourt, De la Méthode grammaticale de Vaugelas [1851], Genève, Slatkine Reprints, 1971, p. 163; C. BADIOU-MonfERRAN, Les Conjonctions de coordination ou "l'art de lier ses pensées" chez La Bruyère, Paris, Champion, 2000, p. 436.

(15) O. Jodogne, Sur la doctrine de Vaugelas, «Vie et langage» n. 127, 1962, p. 520; G. Michaut, La Bruyère, Paris, Boivin, 1936, p. 188.

(16) D. Bouhours, Remarques nouvelles sur la langue françoise, Paris, Sebastien Mabre-Cramoisy, 1676, pp. 84-85; LA ROCHEFOUCAULD, «Réflexions diverses», V, op. cit., p. 210.

(17) A. DE Boisregard, dans H. Sternischa, Deux grammairiens de la fin du XVII siècle: L. Aug. Alemand et Andry de Bois-Regard, Grenoble, SaintBruno/Paris, Armand Colin, 1913, p. 228; LA BruYÈRE, op. cit., II, 28. 
mauvaise presse. Aussi les réalités mixtes ou transitoires, qui sont souvent celles qui séduisent le plus les mentalités modernes, sont-elles désavouées d'une même voix; moralistes et remarqueurs ont en commun une singulière phobie du mélange, assurément symptomatique de la pensée classique: «il faut euiter les vers dans la prose autant qu'il se peut, sur tout, les vers Alexandrins, \& les vers communs»; «Iphis [...] met du rouge, mais rarement, il n'en fait pas habitude. Il est vrai aussi qu'il porte des chausses et un chapeau» ${ }^{18}$. Cette méfiance à l'égard des zones mêlées ou transitoires a souvent été commentée: «l'orientation cardinale de l'esthétique classique, qui aboutit dans son processus [...] au désaveu de l'hybridation»; «un désordre, un trouble fondamentaux, où tout se mêle à tout, où tout se confond avec tout» ${ }^{19}$.

L'art du discernement se traduit encore par une attention particulièrement accusée aux détails et à l'infime. Ce sont de petites choses que décrivent les moralistes comme les remarqueurs; ce ne sont de part et d'autre qu'humbles «minuties» de style ou mouvements intérieurs fugaces et «imperceptibles», à la limite de l'inconscient: «ceux qui n'ont pas le goust de nostre Langue, \& qui ne sçavent ce que c'est qu'un stile exact, se moqueront sans doute de ces minucies»; «l'aversion du mensonge est souvent une imperceptible ambition de rendre nos témoignages considérables» ${ }^{20}$. La critique, de part et d'autre, relève volontiers cet intérêt pour les petites choses: «des petits faits grammaticaux comme l'accord des participes, des détails d'orthographe ou même des manières de prononcer»; «[La Bruyère] ne découvre que des vérités de détail $\gg^{21}$.

Pour décrire leur démarche hyperboliquement analytique, moralistes et remarqueurs convoquent d'un commun accord le modèle méthodologique de l' «anatomie». Cette image transversale, assurément décisive et souvent commentée ${ }^{22}$, se prête aussi bien à une application linguistique qu'à une application morale. Vaugelas envisage d'«esplucher cette façon de parler, se louër de quelqu'vn, \& en faire une anatomie», et La Rochefoucauld, à travers l'imaginaire de la sinuosité obscure - le dragon n'est pas loin -, joue finement, par syllepse, sur le double sens, concret et abstrait, de cour: «faire l'anatomie de tous les replis du cœur» ${ }^{23}$.

Le goût de la bizarrerie

Moralistes et remarqueurs partagent la même aptitude à déceler l'étrangeté insoupçonnée de leur objet d'étude, mœurs ou langue: le mot «bizarre» est un leitmotiv collectif, incontournable sous leur plume. En les lisant, on apprend ainsi à voir ce qu'on ne voyait pas, pour l'avoir trop constamment sous les yeux: le mystère ou le ridicule d'un phénomène qui semblait banal et anodin. L'autorité de l'usage, explique-t-on d'un côté, l'emporte sur celle de la raison: aussi n'est-il pas rare que tel accord ou telle signification ne coïncide pas avec ce qu'on attendrait en bonne logique. L'homme est lui-même un étrange monstre de foire, ajoute-t-on de l'autre côté, et l'adoption d'un point de vue distancié en révèle toutes les lubies puériles

(18) Vaugelas, op. cit., p. 102; La Bruyère, ibid., XIII, 14.

(19) W. Ayres-Bennett et Ph. Caron, «Préface», dans Les Remarques de l'Académie française sur le "Quinte-Curce" de Vaugelas, Paris, Presses de l'École Normale Supérieure, 1996, p. 26; J. LAFOND, La Rochefoucauld: Augustinisme et littérature, $3^{e}$ éd., Paris, Klincksieck, 1986, p. 189.

(20) D. Bounours, Doutes sur la langue françoise,

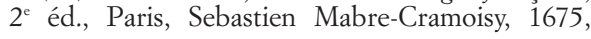

p. 255; La Rochefoucauld, op. cit., maxime 63.

(21) M. Cohen, «Vaugelas et le français de classe», dans Grammaire et style, Paris, Éditions Sociales, 1954, p. 60; TAINE, dans Célébration de La Bruyère, Paris, Valmonde-Trédaniel, 1996, p. 132.

(22) Voir L. VAn DeLFT, Littérature et anthropologie: Nature bumaine et caractère à l'époque classique, Paris, PUF, 1993.

(23) Vaugelas, op. cit., p. 463\%; La RochefouCAULD, op. cit., lettre au P. Thomas Esprit, p. 309. 
et autres contradictions internes. Mais c'est justement ce caractère énigmatique qui stimule la curiosité de l'observateur, et qui le conduit à faire des découvertes: «ce sont des bizarreries de l'usage»; «Quelle bizarrerie! $\gg^{24}$. Les commentateurs signalent parfois cette hypersensibilité à l'étrangeté: «bien faire comprendre tous les degrés et toutes les nuances de la "bizarrerie" de cet usage»; «aussi n'a-t-on pas suffisamment pris la mesure, à ce jour, de la part de l'étrange dans l'esthétique de La Bruyère» ${ }^{25}$.

Mais la bizarrerie appelle-t-elle le scandale ou l'amusement? Ce n'est guère s'avancer que de dire que l'attitude d'un Vaugelas tient davantage des «anomalistes» que des «analogistes» ${ }^{26}$ : c'est-à-dire que l'irrégularité et l'exception ont aussi leurs charmes à ses yeux, quand d'autres idéaliseraient la parfaite prévisibilité des règles. Repérant incidemment une bizarrerie ponctuelle, il s'en délecte d'abord avec curiosité, avant de tenter d'en rendre raison. Il y a aussi de cela en La Bruyère, alors même qu'il aime railler les ridicules des collectionneurs obsédés par la pièce manquante: c'est en quelque sorte un collectionneur de collectionneurs, qui a la particularité de rechercher uniquement les belles pièces au lieu de viser l'exhaustivité. Quoi qu'il en dise explicitement, on sent bien en le lisant qu'au fond il s'amuse beaucoup du spectacle étrange que constitue le monde: on devine son sourire, indulgent autant que railleur, quand il repère chez l'un de ses contemporains telle lubie inédite, qu'il s'empresse d'ajouter à sa galerie. Vaugelas et La Bruyère partagent donc la même sympathie, affichée ou latente, pour les monstres: «cette façon de parler s'attaquer à quelqu'vn, pour dire attaquer quelqu'vn, est tres-estrange \& tres-Françoise tout ensemble»; «cet autre aime les insectes; il en fait tous les jours de nouvelles emplettes: c'est surtout le premier homme de l'Europe pour les papillons $\rangle^{27}$. Cette attitude de collectionneur amusé est parfois commentée: «rien n'égale la satisfaction de Vaugelas lorsqu'il découvre une "belle et curieuse exception" aux règles qu'il s'efforce d'établir»; «le grotesque, l'excentrique ne sont pas exposés comme des vices, mais comme des curiosités qui intéressent l'écrivain ${ }^{28}$.

Protée n'est-il pas le monstre des monstres? De fait, l'attention de Vaugelas et de La Bruyère est prioritairement attirée par tout ce qui est mouvant, instable, insaisissable. La difficulté de fixer un référent ondoyant n'a pas échappé à la sagacité des critiques: «chez Vaugelas et ses commentateurs, l'impossibilité de trancher est souvent manifeste»; «on y voit des caractères hésiter (littéralement) entre deux ou plusieurs dénominations, comme si l'image n'admettait pas de se laisser fixer dans un cadre» ${ }^{29}$. D'où le lien entre la forme brève et l'imaginaire baroque: «les remarques détachées ont aussi tendance à examiner [...] la variation, l'instabilité et le changement dans la langue»; «la matière entre toutes ondoyante et fuyante des moralistes» ${ }^{30}$.

De fait, la mise au point ne semble pas se faire sur certains référents, qui se dérobent sous le regard analytique, et sur lesquels on ne parvient pas à trancher nette-

(24) A. DE Boisregard, dans Commentaires sur les remarques de Vaugelas, éd. J. STREICHER, Paris, Droz, 1936, p. 922; LA BRUYĖe, op. cit., XI, 76.

(25) K.A. Otт, art. cit., p. 83; B. RoukHOMOvsky, "Les Caractères" de La Bruyère ou la cérémonie burlesque: Du théâtre du monde au monde à la renverse, thèse, Université Paris X, 1997, p. 44.

(26) Voir J.-C. Chevalier, Histoire de la syntaxe: Naissance de la notion de complément dans la grammaire française [1968], Paris, Champion, 2006, pp. 273-274.

(27) Vaugelas, op. cit., p. 472; La Bruyère, op. cit., XIII, 2.

(28) A. FrançoIs, La Grammaire du purisme et
l'Académie française au XVIII siècle, Paris, Société Nouvelle de Librairie et d'Édition, 1905, p. 133; F. Gray, La Bruyère amateur de caractères, Paris, Nizet, 1986, p. 68.

(29) G. SIOUFF, Vaugelas et la notion de variation, «Revue des langues romanes», n. 1, 1997, p. 128; M. Escola, La Bruyère, Paris, Champion, 2001, t. I, p. 227.

(30) W. Ayres-BenNeTt, Observations et remarques sur la langue française: Histoire d'un genre, «La Licorne» n. 19, 1991, p. 14; L. VAN DELFT, Les Moralistes: Une apologie, Paris, Gallimard, 2008, p. 51. 
ment. L'idéal méthodologique de la fixité se confronte alors à la souplesse constitutive du réel, qui ne se laisse pas aisément réduire à des cases bien délimitées, et qui garde comme l'empreinte imaginaire des poètes de l'inconstance. C'est quand les repères usuels s'effritent que la parole du moraliste ou du remarqueur trouve sa pleine légitimité: «par quel moyen est-ce donc que l'on peut s'esclaircir de cét Vsage, quand il est douteux \& inconnu?»; «comment le fixer, cet homme inquiet, léger, inconstant, qui change de mille et mille figures?» ${ }^{31}$.

\section{Rencontres éparses}

Les convergences internes ne se limitent pas aux grands thèmes transversaux décrits ci-dessus: il faudrait compléter le dossier par une série d'éléments plus ponctuels, plus anecdotiques, dont on ne fera ici qu'ébaucher brièvement l'inventaire. Entre moralistes et remarqueurs, les images se recoupent parfois: «la Cour est comme un magazin, d'où nostre langue tire quantité de beaux termes pour exprimer nos pensées»; «il y a un certain nombre de phrases toutes faites, que l'on prend comme dans un magasin» ${ }^{32}$. Les arguments coïncident: «il y en a qui disent encore des soins inutils, pour, des soins inutiles, comme si on disoit inutil au masculin, \& inutile au feminin»; «au contraire de vil, vile, subtil, subtile, selon leur terminaison masculins ou féminins» $»^{33}$. Les préceptes convergent: «l'on ne parle que pour se faire entendre»; «l'on n'écrit que pour être entendu» ${ }^{34}$.

Incidemment, on pourrait même élargir la question à d'autres formes de discours grammaticaux que les remarques proprement dites, car l'influence de la Grammaire générale et raisonnée, pour être ponctuelle, n'en reste pas moins très reconnaissable dans Les Caractères: «il y a trois opérations de notre esprit: CONCEVOIR, JUGER, RAISONNER»; «Qui doute que les enfants ne conçoivent, qu'ils ne jugent, qu'ils ne raisonnent conséquemment?» ${ }^{35}$.

\section{Convergences externes}

\section{Une respectabilité en péril}

Quelque légitime passion qu'on éprouve pour les moralistes ou les remarqueurs, on a toujours un peu mauvaise conscience, au fond de soi-même, de s'intéresser à eux en les prenant au sérieux. Cet intérêt intellectuel ne va jamais tout à fait de soi: même fugace, même aussitôt écarté, le malaise est là, initialement, qu'on l'assume ou qu'on l'ignore. Malgré tous les apports de la recherche, subsiste toujours un dédain collectif pour ceux que le grand public, parce qu'il se garde bien de les lire, considère spontanément comme des donneurs de leçons, dogmatiques, étroits d'esprit et vétilleux. L'Université elle-même n'est pas tout à fait épargnée par ce climat de méfiance ou d'hostilité. Car de savantes plumes ont plus d'une fois affirmé qu'un moraliste ou un remarqueur n'avait pas de «système», pas de «vue d'ensemble», pas de «théorie», ce qui, au pays du cartésianisme triomphant, signifie à peu près une absence de pensée digne de ce nom. Il n'y a de cohérence et de profondeur que dans la continuité, s'obs-

(31) Vaugelas, op. cit., préface, IV; La BRuYÈre, op. cit., XIII, 19.

(32) Vaugelas, ibid., préface, II; La Bruyère, ibid., VIII, 81.

(33) T. CoRneIlle, dans Commentaires sur les remarques de Vaugelas cit., p. 936; LA BRUYÈRE, ibid., XIV, 73.

(34) Bouhours, Doutes sur la langue françoise cit., p. 183; La Bruyère, ibid., I, 57.

(35) A. Arnauld et C. LanCElot, Grammaire générale et raisonnée [1660], Paris, Allia, 2010, p. 47; LA BRuYÈRE, ibid., XI, 58. 
tinent à croire certains auteurs, poursuivant ainsi l'éternel «procès», sans parvenir à dépasser le scandaleux éparpillement de la forme brève: «Vaugelas [...] ne s'efforce en rien de donner quelque rigueur à un système en formation»; «[La Bruyère] étranger à toute ambition philosophique, n'ayant nullement l'idée de bâtir un système» ${ }^{36}$.

Les spécialistes des moralistes ou des remarqueurs sont donc d'abord leurs avocats, et les étudier, c'est toujours, peu ou prou, réaffirmer avec passion et véhémence leur pleine respectabilité, alors qu'on n'observe rien de tel pour la plupart des «grands auteurs» reconnus par la postérité: «la grammaire des "remarques" a été relativement négligée jusqu'à ces derniers temps»; «le personnage [du moraliste], quand il n'est pas tout bonnement relégué aux oubliettes ou même diabolisé, demeure profondément méconnu» ${ }^{37}$.

Aujourd'hui encore, un travail savant - essai ou édition critique - commence donc souvent par une tentative de légitimation du propos: ce qui implique bel et bien, en creux pour ainsi dire, qu'on est loin d'en avoir fini avec le désaveu collectif. Travailler sur les moralistes ou sur les remarqueurs, c'est d'abord s'excuser de le faire, comme si c'était en soi répréhensible: «le nom de Vaugelas étonne peut-être ici»; «les lecteurs de La Rochefoucauld que furent Stendhal et Nietzsche suffiraient à prouver que les bons esprits n'ont pas cru que ce fût là une œuvre à réserver à l'usage mondain $\gg^{38}$.

\section{Le problème de la norme}

Si moralistes et remarqueurs ont mauvaise presse, c'est avant tout parce qu'ils sont perçus comme exprimant une norme, dans leurs domaines respectifs. Ce grief est pourtant parfaitement anachronique, ne fût-ce que pour des raisons de pure terminologie, sans même parler des modèles scientifiques ${ }^{39}$. Or, chacun peut aisément constater qu'une méfiance très forte imprègne les mentalités modernes à l'égard des donneurs de leçons. Le discrédit des «moralisateurs» les plus dogmatiques entraîne donc dans son sillage le cortège des moralistes, pourtant autrement subtils et nuancés. De même, au nom de l'idéal scientifique de neutralité absolue qui prétend fonder la linguistique, d'aucuns s'obstinent à affirmer qu'il y aurait une différence radicale entre l'approche ouvertement normative du «grammairien» et l'approche purement descriptive du «linguiste» ${ }^{40}$. C'est oublier qu'un idéal est par définition toujours pourchassé, et toujours inaccessible.

On s'attendrait à ce que les spécialistes des moralistes et des remarqueurs prissent leurs distances avec ce discours ambiant de désaveu du ton dogmatique; ils semblent au contraire, dans bien des cas, l'avoir intégré comme une évidence a priori, ce que suggère le malaise qu'on perçoit parfois sous leur plume. Certes, les descriptions les plus objectives reconnaissent sans ambiguïté l'orientation normative du propos: «'univers de discours est principalement celui de la grammaire prescriptive»; «[les

(36) J.-C. Chevalier, op. cit., p. 472; PrévostPARADOL, Etudes sur les moralistes français, $8^{\mathrm{e}}$ éd., Paris, Hachette, 1895, p. 142.

(37) W. Ayres-BEnNETT, Observations et remarques sur la langue française cit., p. 1; L. VAN DELFT, Les Moralistes: Une apologie cit., pp. 18-19.

(38) G. Siouffi, Penser le langage à l'Áge classique, Paris, Armand Colin, 2010, p. 33; J. LAFOND, op. cit., p. 9 .

(39) Selon P. Dumonceaux, «Vaugelas, ignorant très probablement le mot, n'a pu avoir la conscience linguistique de la Norme» (Le bon usage est-il une norme?, «Textes et langages» n. 12, 1986, p. 38); cf.
Gilles Siouffi: «c'est un terme absent du métalangage linguistique du XVII ${ }^{\circ}$ siècle» ( $\ll$ La norme lexicale dans les Remarques sur la langue française de Vaugelas», dans G. SioufFI et A. STEUCKARDT (dir.), La Norme lexicale, Montpellier, Dipralang, 2001, p. 60).

(40) André Martinet établit explicitement un parallèle avec les questions morales: «La difficulté qu'il y a à dégager la linguistique scientifique de la grammaire normative rappelle celle qu'il y a à dégager de la morale une véritable science des mœurs» (Éléments de linguistique générale, Paris, Armand Colin, 1970, p. 6). 
moralistes] se voient obligés d'assumer la position théocratique du législateur ou du juge, une posture de maîtrise ouvertement déclarée» ${ }^{41}$.

Mais bien plus souvent, ce qu'on observe, c'est plutôt une tendance à estomper cet aspect, jugé trop réducteur, de l'entreprise des moralistes et des remarqueurs, comme s'il était en soi coupable, et comme s'il fallait l'atténuer pour rendre à ces auteurs leur pleine respectabilité. Ce déni de normativité peut prendre plusieurs formes. On peut se contenter d'insister sur la grande prudence du ton de l'auteur dont on traite, en montrant que, loin de parler avec assurance et dogmatisme, il atténue son propos par le jeu nuancé de la modalisation, ou par le recours aux degrés de comparaison, promouvant ainsi une normativité scalaire: «la récurrence de l'adverbe mieux [...] est à cet égard significative d'une axiologie scalaire et non bi-polaire»; «le $\mathrm{XVII}^{\mathrm{e}}$ siècle français présente [...] une tendance particulièrement forte vers la maxime non catégorique» ${ }^{42}$.

L'orientation normative peut aussi être présentée comme un élément périphérique de l'activité du moraliste ou du remarqueur, qu'on ne songe pas à contester, mais dont on relativise la portée: «later readers of the remarqueurs abridged and simplified their work, making it appear much more dogmatic than in reality»; «l'écrivain qui traite des mœurs et (ou) s'adonne à l'analyse, en ne s'interdisant pas de rappeler des normes» ${ }^{43}$.

On peut par ailleurs user du contrepoint, c'est-à-dire présenter l'auteur spécifique qu'on étudie comme moins normatif que ses concurrents. La normativité, alors, n'est pas réduite, mais déplacée: on la perçoit et on la désavoue en elle-même, mais on estime qu'elle intervient ailleurs. La fascination - toujours légitime - pour un auteur donné, qu'on rêve comme exceptionnel, peut alors conduire à une forme d'injustice pour d'autres auteurs, traités comme des repoussoirs: «Andry semble plus tolérant que des remarqueurs tels que Vaugelas ou Bouhours»; «Chamfort est cet étrange "moraliste" qui se garde de moraliser» ${ }^{44}$.

Il est encore d'usage d'identifier, chez les moralistes comme chez les remarqueurs, des précurseurs de certaines inflexions modernes des sciences humaines. C'est une façon de mettre en évidence l'intérêt qu'ils peuvent présenter à nos yeux, aujourd'hui encore, au prix d'un anachronisme délibéré. Mais c'est aussi une voie pour réduire la part de normativité chez ces auteurs, et donc un hommage inconsciemment rendu à l'idéologie dominante, puisque le sociologue ou le sociolinguiste est enclin à se présenter lui-même, à tort ou à raison, comme un observateur impartial: «their approach may be thought of as socio-linguistic avant la lettre»; «anthropologue avant la lettre» ${ }^{45}$.

Certains commentateurs, entraînés par leur enthousiasme, vont même parfois jusqu'à nier purement et simplement la part de normativité indissociable des œuvres qu'ils analysent: «Vaugelas n'a point posé ni proposé de lois; il a exprimé des opinions, il a constaté des faits, et il a donné des conseils»; «i moralisti non prescrivono ma si limitano a descrivere ${ }^{46}$.

(41) Ph. Caron, op. cit., p. 396; F. JaOUËN, De l'Art de plaire en petits morceaux: Pascal, La Rochefoucauld, La Bruyère, Saint-Denis, Presses Universitaires de Vincennes, 1996, p. 159.

(42) W. Ayres-BennetT et Ph. Caron, op. cit., p. 19; C. SCHAPIRA, La Maxime et le discours d'autorité, Paris, SEDES, 1997, p. 83.

(43) W. Ayres-BennetT, Reading the 'remarqueurs': Changing perceptions of 'classic' texts, «Historiographia linguistica», 2006, XXXIII-3, p. 295 ; L. VAn Delft, Le Moraliste classique: Essai de défi- nition et de typologie, Genève, Droz, 1982, p. 108. (44) M. SEIJIDO, Etude critique d'un remarqueur de la fin du XVII siècle: Nicolas Andry de Boisregard, thèse, Université Aix-Marseille I, 2001, p. 228; F. JEANSON, «Le moraliste grandeur nature», dans Lignes de départ, Paris, Seuil, 1963, p. 103.

(45) W. Ayres-BENNETT, Reading the 'remarqueurs', art. cit., p. 271; L. VAN DELFT, Littérature et anthropologie cit., p. 28.

(46) F. BRunETIĖRE, «Vaugelas et la théorie de l'usage», Études critiques sur l'bistoire de la littéra- 
Il faut toutefois signaler une différence importante entre remarqueurs et moralistes sur ce point. On s'accorde généralement à considérer que de Vaugelas aux remarqueurs tardifs, le discours grammatical passe graduellement de la souplesse à l'intransigeance. Au contraire, on identifie une atténuation progressive de la part de prescription dans l'analyse morale. L'évolution des discours n'est donc pas parallèle, mais diamétralement opposée selon le domaine traité, ce qui peut surprendre. Au cours du XVII ${ }^{\mathrm{e}}$ siècle, les remarqueurs sont de plus en plus dogmatiques, alors que les moralistes le sont de moins en moins:

Si l'usage de Molière contredit une norme, c'est rarement la norme de Vaugelas, mais bien plus la norme de ses commentateurs ultérieurs, Bouhours, Thomas Corneille, Andry de Boisregard, l'Académie, tous ces doctes qui, à partir des années 1670 [...], commentent et durcissent notablement les Remarques, préparant ainsi la voie au purisme grammatical du XVIII siècle.

[...] on s'imagine trop souvent que le moraliste classique conseille et prescrit, joue les prédicateurs laïques, en un mot moralise. Si cette attitude se rencontre encore dans la première moitié du siècle, elle est pratiquement absente de la littérature d'après $1660^{47}$.

\section{Particularismes français}

Les genres respectifs des réflexions morales et des observations sur la langue ont en commun d'être souvent perçus et présentés comme typiquement français. Un imaginaire de l'esprit national se manifeste alors, inévitablement superposé à la description objective des traditions culturelles: il y aurait en France une pensée spécifique, s'exprimant par une forme spécifique. De telles vues reposent sur des constats que chacun peut aisément faire: les mêmes Français qui entretiennent une certaine tradition de la «pensée du jour» ont conservé une idolâtrie tout à fait singulière de leur langue, même - ou surtout? - quand ils la malmènent ${ }^{48}$. Les vétilles orthographiques, en particulier, passionnent autant qu'elles agacent: elles font l'objet d'une forme de vénération exaltée qui semble objectivement disproportionnée, pour peu que l'on prenne du recul, et qui tient sans doute à leur énigmatique complexité. Même si les rubriques grammaticales d'antan semblent avoir déserté les colonnes des journaux, les forums de discussion sur Internet ont clairement pris le relais. Les commentateurs s'accordent donc à étendre à l'ensemble d'une nation l'attitude spécifique des moralistes ou des remarqueurs: «la forme des remarques détachées [...] semble, au moins à des yeux étrangers, typiquement française»; «rien de mieux approprié que ces petits genres, si spécialement français, à une société de femmes, à un auditoire de salon» ${ }^{49}$.

\section{Empirisme de la démarche}

Moralistes et remarqueurs sont généralement présentés comme de simples observateurs des faits, sans réelle volonté de théorisation: «le contenu a tendance à être axé sur les données (data-orientated) plutôt que sur une théorie quelconque (theoryorientated)»; «un "moraliste" n'est pas non plus un philosophe au sens moderne du terme, pour qui la morale serait matière à réflexion spéculative» ${ }^{50}$.

ture française, $7^{\mathrm{e}}$ série, $2^{\mathrm{e}}$ éd., Paris, Hachette, 1905 , p. 52; C. Rosso, Inventari e postille, Pise, Goliardica, 1974 , p. 187.

(47) N. Fournier, La langue de Molière et le bon usage de Vaugelas dans "Les Femmes savantes", «L'Information grammaticale» n. 56, 1993, p. 20; J. LAFOnD, Moralistes du XVII siècle cit., préface, p. XIV.

(48) «Qu'on se le dise, notre langue n'est pas un idiome comme les autres, c'est une merveille du monde. Les Français lui vouent un véritable culte, un attachement orgueilleux et patriotique» (F. DE Closets, Zéro faute: L'Orthographe, une passion française, Paris, France Loisirs 2010, p. 33).

(49) W. Ayres-BennetT, Observations et remarques sur la langue française cit., p. 1; J. BouRDEAU, La Rochefoucauld, Paris, Hachette, 1895, p. 79 .

(50) W. Ayres-Bennett, ibid., p. 13; B. ParmenTIER, op. cit., p. 7. 
Aussi les mots «empirisme» ou «expérience» reviennent-ils régulièrement sous la plume des commentateurs. Cet empirisme peut être pris en bonne ou en mauvaise part; on peut y lire la carence d'une doctrine, mais aussi la crédibilité d'un constat ou d'un témoignage: «Vaugelas n'est qu'un empirique»; «les Maximes, qui illustrent une philosophie de l'expérience» ${ }^{51}$.

Les organes sensoriels qui incarnent métonymiquement cette démarche d'observation des faits, il est vrai, ne sont pas les mêmes pour le moraliste et pour le remarqueur: celui-là promène ses yeux autour de lui, celui-ci laisse traîner ses oreilles. Mais ils ont une fonction symbolique analogue: «observateur attentif et patient, Vaugelas avait passé sa vie à écouter la France»; «c'est bien ce qu'a fait La Rochefoucauld pendant toute sa vie: observer» ${ }^{52}$.

\section{Matière et manière}

L'idée que la forme brève est adoptée parce qu'elle conviendrait tout particulièrement à l'objet traité est peut-être le plus stimulant des topoï que véhicule le discours critique sur les moralistes ou sur les remarqueurs. Ces derniers, dit-on volontiers, seraient naturellement et nécessairement portés vers cette forme éclatée, par une manière de contrainte interne: ce serait la seule forme vraiment adaptée pour décrire la mouvance permanente de la psychologie humaine ou les hésitations éparses et ponctuelles de l'usage linguistique. La forme brève, en quelque sorte, ressemblerait à la question abordée, elle en reproduirait le caractère instable, diffus ou parcellaire, elle multiplierait les éclairages les plus divers pour saisir les mille facettes d'un référent infiniment complexe: «la matière traitée par Vaugelas, à savoir les finesses et les idiotismes de l'usage du français, ne s'accordait pas avec un ordre plus rigide»; «tout se passe donc comme si la diversité et la contradiction de l'expérience humaine ne pouvaient se formuler qu'au moyen d'une écriture ponctuelle, fragmentaire» ${ }^{53}$.

De fait, l'éclatement du discours est le trait le plus immédiatement visible de l'écriture des moralistes comme des remarqueurs, au point d'en être quasi définitoire: «volumes of generally short observations or remarks on points of doubtful usage»; «la forme du fragment (qui a partie si étroitement liée avec l'écriture des moralistes) $\gg^{54}$.

La critique, de part et d'autre, décrit l'impression que chacun peut éprouver en lisant les moralistes ou les remarqueurs: celle d'une harmonie accomplie entre le propos et la présentation - à supposer encore qu'on puisse les dissocier mentalement, ce qui ne va certainement pas de soi. Le recours à la forme brève ne saurait être aléatoire: car la littérarité des moralistes comme la crédibilité des remarqueurs seraient alors sérieusement ébranlées. La plus grande réussite de ces auteurs, c'est finalement de créer en nous la conviction inébranlable qu'ils ne pouvaient pas dire autrement ce qu'ils ont dit: «la forme même - rhapsodique par essence - des observations détachées est particulièrement propice à la discussion de l'usage douteux»; «c'est toujours pour décrire au plus près l'homme que l'on retrouve, avec la forme du fragment, cette composition désinvolte, ce désordre du plan» ${ }^{55}$.

(51) C.-A. SainTe-Beuve, «Vaugelas», dans Nouveaux lundis, t. VII, Paris, Michel Lévy Frères, 1866, p. 357; A. MAZÈre, La Rochefoucauld: Le Duc rebelle, Paris, Le Croît Vif, 2007, p. 266.

(52) E. Moncourt, op. cit., p. 22; G. Minois, op. cit., p. 18.

(53) W. Ayres-BennetT, Observations et remarques sur la langue française, art. cit., p. 8; J. STAROBINSKI, La Rochefoucauld et les morales substitu- tives, «La Nouvelle Revue française» n. 163, 1966, p. 22.

(54) W. Ayres-Bennett, Reading the 'remarqueurs' cit., p. 263; L. VAN DELFT, Les Moralistes: Une apologie cit., p. 32.

(55) W. Ayres-Bennett, Les ailes du temps et la plume du 'remarqueur': La tradition puriste au XIX siècle, «Romantisme», n. 86, 1994, p. 33; L. VAN Delft, Le Moraliste classique cit., p. 260. 


\section{Conclusion}

La Rochefoucauld et Bouhours ou La Bruyère et Vaugelas sont moins dissemblables que ne le suggère la coupure universitaire entre les disciplines: des parentés précises se manifestent dans leurs pensées et leurs méthodes respectives, au point qu'on s'étonne que le rapprochement ne soit pas fait plus souvent. Parallèlement, les commentaires des spécialistes respectifs des moralistes et des remarqueurs se rejoignent très nettement, contribuant ainsi à esquisser ce qui n'est encore que virtuel: une description transversale de la forme brève au XVII ${ }^{\mathrm{e}}$ siècle, tous domaines confondus. Chemin faisant, l'auteur de ces lignes a été contraint de prendre un certain recul à l'égard d'un discours critique collectif, dont il est lui-même pleinement partie prenante: on aura compris que ce recul, nécessaire à la prise de conscience et à la mise en évidence des convergences «externes», n'est en rien un désaveu. Le son de la recherche sur les formes brèves, si l'on ose dire, est stéréophonique. Du dossier ainsi esquissé, on ne tirera pas la conclusion que chacun doit tendre à dédoubler sa spécialisation: ce serait évidemment disproportionné. Mais peut-être que chacun gagnerait à se montrer plus attentif à ce qui se pratique dans un domaine de recherche bien distinct mais rigoureusement parallèle: la duplication effective des discours critiques leur assure finalement une manière de confirmation indirecte, tout en suggérant des pistes nouvelles. Et peut-être que les démarches respectives des moralistes et des remarqueurs, qui gardent leur part de mystère, s'éclaireront davantage à être ainsi confrontées. 